\title{
Un exemple de valorisation de la France centrale par le rail : le « Tacot » à Cosne d'Allier, 1887-1950
}

Roger Courtaud

\section{OpenEdition}

\section{Journals}

Édition électronique

URL : https://journals.openedition.org/rhcf/2080

DOI : 10.4000/rhcf.2080

\section{Éditeur}

Rails \& histoire

Édition imprimée

Date de publication : 2 mai 2002

Pagination : 342-359

ISBN : 00996-9403

ISSN : 0996-9403

\section{Référence électronique}

Roger Courtaud, « Un exemple de valorisation de la France centrale par le rail : le « Tacot » à Cosne d'Allier, 1887-1950», Revue d'histoire des chemins de fer [En ligne], 24-25 | 2002, mis en ligne le 21 avril 2015, consulté le 22 avril 2022. URL : http://journals.openedition.org/rhcf/2080 ; DOI : https://doi.org/ $10.4000 /$ rhcf.2080 
Roger COURTAUD

Association "Mémoire en pays cosnois »

\section{Un exemple de valorisation de la France centrale par le rail : le " Tacot» à Cosne d'Allier, 1887 - 1950}

\section{Mémoire du pays cosnois : qui sommes-nous ?}

Un tout jeune club d'histoire locale qui se penche sur le passé de Cosne d'Allier et de ses environs immédiats, petit ensemble communautaire de 3500 habitants, situé $30 \mathrm{~km}$ au nord-est de Montluçon.

Nos activités à ce jour : un bulletin annuel et quatre expositions rétrospectives régulièrement pérennisées par une plaquette.

Le «Tacot», ce petit train qui a animé notre bocage bourbonnais pendant 63 ans a, tout naturellement, été l'un des premiers sujets abordés.

Cette initiative a suscité l'enthousiasme de la population locale au sein de laquelle se comptent encore quelques survivants et surtout de nombreux descendants des anciens employés de l'entreprise. Quelques passionnés du rail, de passage dans notre région, se sont également intéressés à notre présentation.

Nos efforts pour faire revivre ce valeureux petit chemin de fer étaient ainsi déjà récompensés ; mais nous sommes particulièrement honorés que l'AHICF ait eu l'obligeance de les prendre aussi en considération.

Mademoiselle Fayolle, notre présidente, Monsieur Beaudonnet, vice-président, et votre serviteur, choisi comme intervenant en raison de ses affinités innées avec les chemins de fer dits « secondaires », plus particulièrement avec le réseau du Centre des Chemins de fer économiques, avons essayé d'analyser la valorisation que notre pays doit à ce fameux « Tacot».

\section{Présentation de Cosne d'Allier}

Qu'était donc notre commune, alors appelée « Cosne sur l'CEil », du nom de l'une des deux rivières qui baignent la localité, avant que les rails ne l'atteignent? Cosne, qui signifie « confluent » en gaulois, est devenue un carrefour naturel. 
Ainsi le conseil municipal de notre petite ville pouvait-il faire état de la présence de neuf embranchements de routes lors des délibérations qui, de 1875 à 1882, précédèrent le choix des tracés ferroviaires.

La vie économique y était active de longue date : scieries, fabriques de draps, de chandelles en étaient des aspects. Mais ce sont surtout les foires, liées à l'activité agricole, qui faisaient la réputation de la cité, depuis des siècles.

\section{Naissance du rail en pays cosnois}

Le chemin de fer s'est assez rapidement développé en Bourbonnais puisque, dès 1851-1854, la jonction Bec d'Allier-MoulinsClermont était réalisée. Les lignes Saint-Germain-des-Fossés-Roanne, Montluçon-Moulins, Montluçon-Bourges maillaient le réseau en 1861.

Mais le centre du département, le «bocage Bourbonnais », restait tout à fait isolé. C'est pourquoi le conseil général de l'Allier décidait, en 1865, de mettre à l'étude un réseau ferré départemental et le projet de l'Allier devait être le premier soumis à la ratification des chambres.

En 1859 déjà, le conseil municipal de Cosne soulignait que la récente création de la voie ferrée Moulins-Commentry-Montluçon, dont Villefranche d'Allier était la gare la plus proche, située à neuf kilomètres de Cosne, favorisait le développement des marchés.

En 1875, le sous-préfet de Montluçon proposait deux tracés pour le chemin de fer devant desservir le département de l'Allier, selon un axe nord-sud, de Sancoins, dans le Cher, à Lapeyrouse, au seuil du Puy-de-Dôme.

Comme l'un était suggéré par Buxières-les-Mines et l'autre par Cosne, le conseil municipal déclarait à propos du tracé concernant la localité : « c'est la vie qui nous a manqué jusqu'à présent pour le développement de notre commerce et de nos industries. » Et, d'emblée, il était proposé de livrer gratuitement tous les terrains nécessaires pour la confection du tracé sur tout le parcours du territoire de la commune.

Quoique la ligne Sancoins-Lapeyrouse fût déclarée d'intérêt général dès 1878 , car soi-disant jugée d'importance stratégique, la déclaration d'utilité publique tarda à être prononcée.

En 1884 seulement, une décision administrative analogue fut prise au bénéfice de la ligne d'intérêt local Moulins-Cosne.

Ainsi était amorcée l'étoile ferroviaire qui allait marquer si profondément notre petite ville (fig. 1). Le 13 octobre 1887, Cosne était reliée à Moulins, puis le 4 novembre 1891 à Sancoins et à Villefranche d'Allier ; Lapeyrouse n'était atteinte que le 24 octobre 1892. 


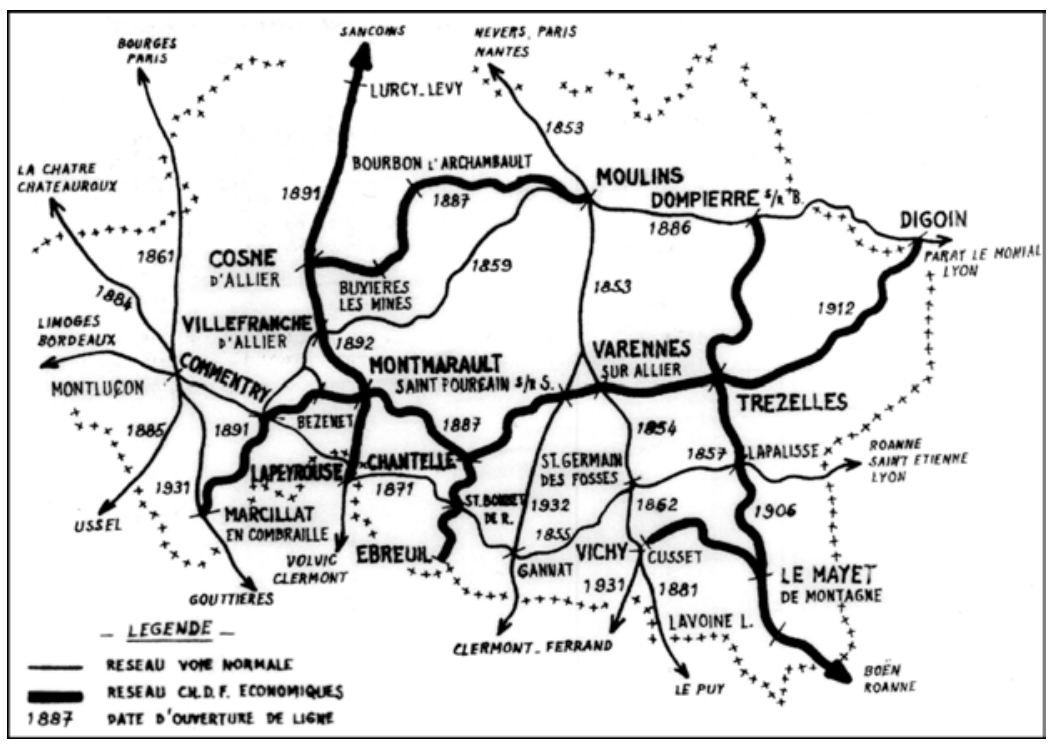

Source : C.R.D.P. Clermont-Ferrand

Figure 1 : Réseau ferré en Bourbonnais : extension maximale (1932).

(c) R. Courtaud

Soulignons que François Mercier, technicien autodidacte né à Tronget en 1858, à vingt kilomètres de Cosne, fit ses premières armes de bâtisseur d'infrastructures ferroviaires sur le tronçon SaintHilaire-Cosne de la ligne de Moulins.

Les voies étroites du Centre, mais aussi Carhaix-Rosporden, Orange-Buis-les-Baronnies, et parmi nos plus belles lignes à voie normale : Saint-Claude-Morez, Bort-les-Orgues-Neussargues, Nice-Coni, devaient ensuite marquer la carrière exceptionnelle de ce Bourbonnais au service du rail.

\section{Des choix fondamentaux}

C'est à la Société générale des chemins de fer économiques, considérée comme spécialiste en la matière, que le département de l'Allier confia la réalisation, puis l'exploitation de ses lignes.

Rendons hommage à nos voisins du Cher, dont les choix intelligents allaient permettre d'établir ce magnifique réseau du Centre (fig. 2).

En retenant la voie métrique, déjà adoptée dans l'Allier, puis en confiant l'exploitation à la Société économique, c'est un ensemble cohérent de $763 \mathrm{~km}$ de lignes, d’intérêt général ou local, qui allait s'étirer sur les deux départements dont l'objectif de faciliter leurs échanges fut ainsi pleinement atteint. 


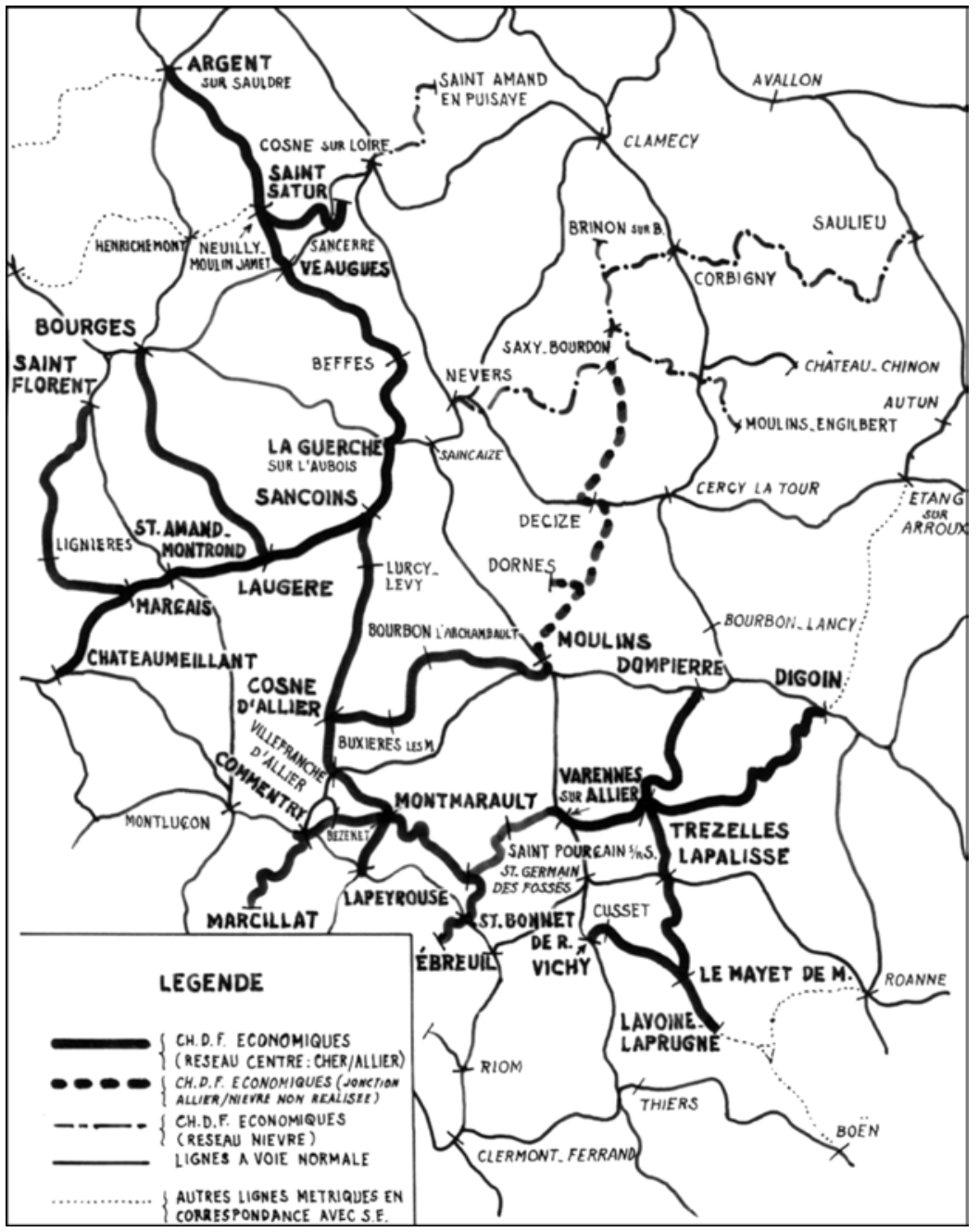

Source : Carte du Réseau du Centre de la S.E., Archives départementales de I'Allier

Figure 2 : Société générale des chemins de fer économiques (bureau du Centre : Cher, Allier, et Nièvre).

${ }^{\circ}$ R. Courtaud 
Pour ne citer que les plus marquants, ce sont les produits agricoles et notamment d'élevage qui s'échangeaient, par trains complets les jours de foire, les ressources forestières, les réalisations de nombreuses industries, si actives alors, comme les porcelaineries, les plâtreries, les fours à chaux, les briqueteries.

Les wagons issus de la cimenterie de Marseilles-lesAubigny-Beffes transitaient nombreux par Sancoins-Lapeyrouse.

A noter que cette industrie permet, de nos jours, le maintien d'un vestige du réseau du Centre, à voie normale, après avoir été à quatre files de rails de 1938 à 1950, entre La Guerche et Beffes.

\section{Le charbon}

Quant au charbon, encore extrait actuellement à Buxières-lesMines pour quelques mois seulement, il était transporté avant la Première Guerre mondiale jusqu'à Blois et Orléans, entièrement par voie métrique !

Les wagons transitaient par Cosne, Sancoins, Argent et Brinonsur-Sauldre, le chemin de fer du Blanc à Argent et les tramways du Loiret.

Cet important gisement de houille et de schistes, dit «Bassin de l'Aumance », qui était desservi par la ligne Moulins-Cosne de SaintHilaire-Gipcy à Buxières, s'est révélé d'une importance capitale pour le réseau.

Avant 1887 et après la suppression de l'Économique en 1950, jusqu'à ces dernières années, le charbon était acheminé par la route jusqu'à la gare de Chavenon, sur la ligne Moulins-Commentry. La période la plus prospère se situa de 1910 à 1919, avec l'extraction de 1054 milliers de tonnes. C'est là que les wagons à double benne culbutante furent le plus sollicités et que le transbordement voie étroite-voie normale de Villefranche d'Allier remplit tout son office, impliquant le passage par Cosne.

\section{Influence du trafic ferroviaire sur la localité de Cosne d'Allier}

Revenons à notre commune, dont la gare, implantée à quelques centaines de mètres du centre ville, a été réalisée en dix-huit mois par des ouvriers recrutés sur place.

A noter que la première route de desserte, rue Henri-Laville actuelle, raccordée à la route de Moulins, avait été prise en charge par la Société économique. 
Sept ans après l'arrivée du premier train, une nouvelle « avenue de la Gare » était tracée, considérée alors avec fierté comme la plus longue du département ! Elle rend hommage, de nos jours, à son promoteur, l'ancien maire, Gabriel Bonnichon.

Voici quelques extraits de Près $d u$ sol, ouvrage de l'écrivain local Emile Guillaumin, qui faillit obtenir le prix Goncourt en 1904 pour son livre La Vie d'un simple (dans lequel la construction de la ligne MoulinsCosne est d'ailleurs évoquée). Ces extraits décrivent l'ambiance autour de la gare de Cosne, avec précision, bien que les noms employés soient à dessein imaginaires.

«La petite place était animée : on attendait le train de Maleville, le chef-lieu, qui devait amener les collégiens en vacances de Pâques. De l'avenue, large et droite, plantée de jeunes arbres, qui reliait la ville à la gare, des groupes débouchaient à chaque instant : vieux messieurs appuyés sur des cannes, bourgeoises en toilette et gamins qui jouaient $[\ldots]$

Des voitures arrivèrent aussi : une charrette de campagne $[\ldots]$ puis un char à bancs verni, puis un coupé de maître [...] enfin, tout à côté de la palissade qui longeait la voie ferrée, près de l'édicule où se lisait l'inscription "lampisterie", se rangea une petite voiture à âne.

Parurent les omnibus de la ville : "Le Lion d'Or", l'hôtel "Mignot", l'hôtel "Belin", [...]

Les conducteurs... couraient boire un canon à la "Buvette de la Gare" [...], qui faisait un tort considérable à l' "hôtel et café de la Gare", établi de l'autre côté. »

Ainsi se présentait cette avenue, qui a toujours belle allure (fig. 3).

Hélas la gare, qui semblait faire un clin d'œil aux usagers en son extrémité, a succombé en 1962 sous la pioche des démolisseurs pour faire place à une maison de retraite et à des lotissements.

Il est certain que tous les commerçants et artisans de Cosne : épiciers en gros, quincailliers, négociants en grains, en engrais, en boissons, mais aussi scieries et distributeurs de matériaux, virent leur activité prospérer grâce au "Tacot» (fig. 4). Les produits agricoles : céréales, fourrages, betteraves, pommes de terre tenaient une place importante. Quant aux bêtes à cornes, aux porcs et aux chevaux, embarqués lors des foires, ils justifiaient par exemple 688 wagons au cours de l'année 1904, soit quelque 75 wagons par foire. 
Revue d'histoire des chemins de fer 24-25 (printemps - automne 2001)
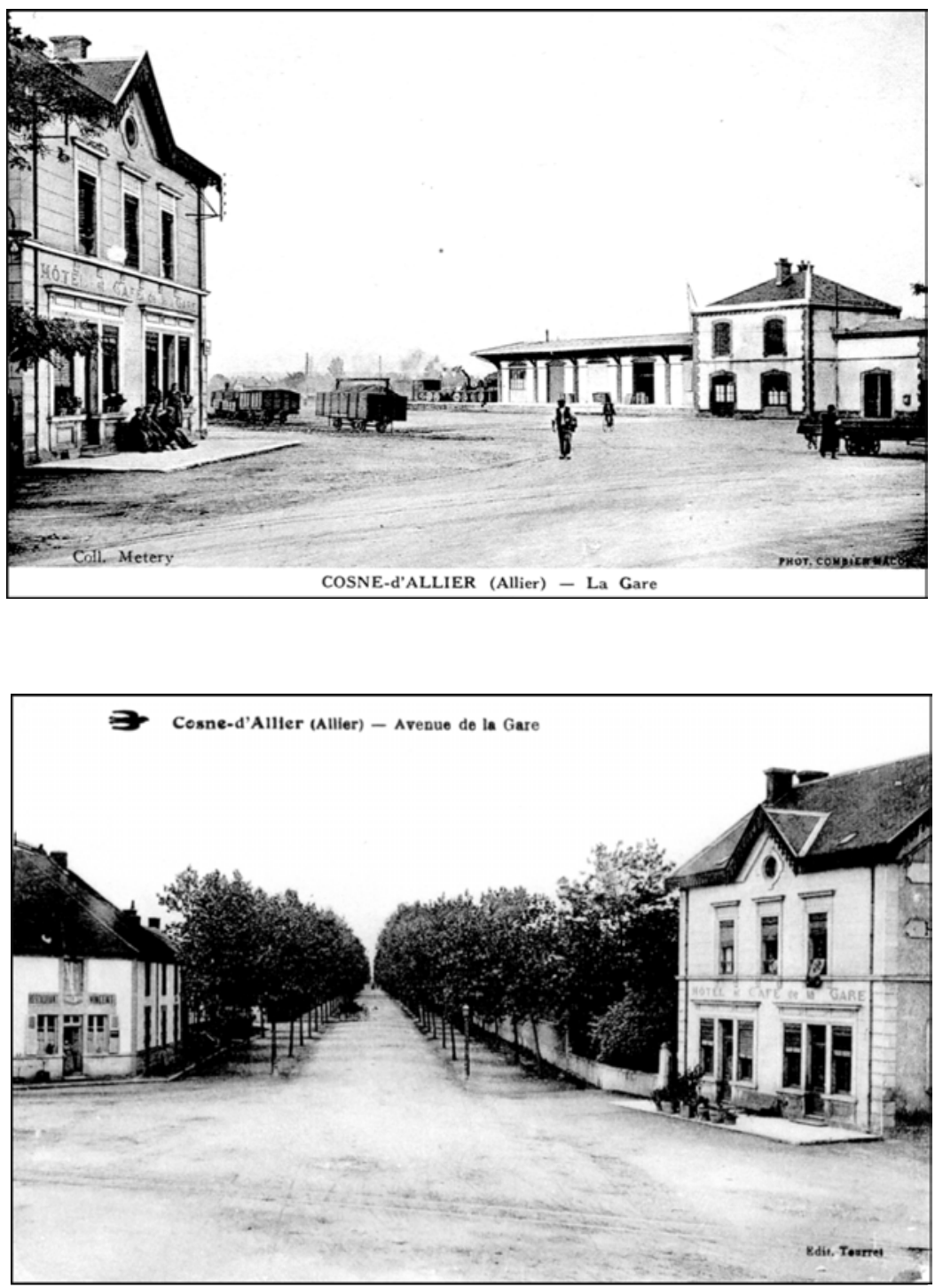

Figure 3 : La gare de Cosne d'Allier et l'avenue de la Gare.

(Haut : 1925. Coll. H. Beaudonnet ; bas : 1930. Coll. P. Alajoinine.) 

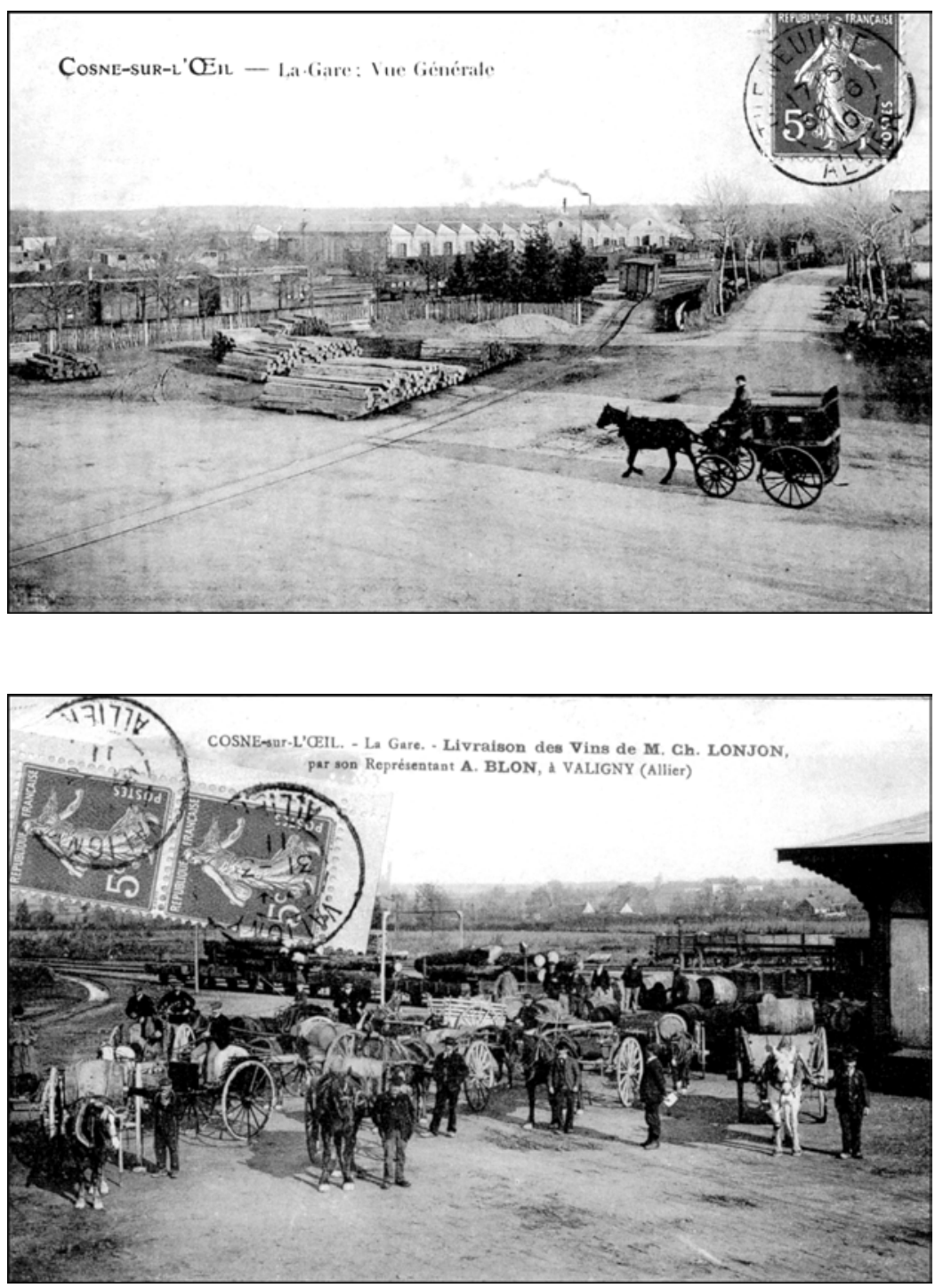

Figure 4 : La gare de Cosne-sur-l'CEil. Trafic des marchandises. (Haut : 1905. Coll. H. Beaudonnet; bas : 1910. Coll. P. Alajoinine.) 
Une anecdote qui montre combien le chemin de fer, «Économique et P.L.M. » réunis, savait assurer promptement un transport de détail, dans les années trente. Chaque semaine, un boucher de Cosne expédiait une corbeille en osier, drapant de la viande dans une grosse toile ficelée, qu'il remettait au train du soir pour Moulins. Le colis était livré le lendemain matin à Paris !

\section{Aperçu sur le trafic " voyageurs"}

En ce qui concerne le trafic voyageurs du début, citons encore Emile Guillaumin : « les habitués : petits bourgeois, gros fermiers, commerçants et curés. En dehors des jours de foire, on n'y voyait guère de paysans, ni d'ouvriers... »

Néanmoins, les trains spéciaux étaient fréquents : pour les jours de foire, certes, mais aussi à l'occasion de fêtes locales ou lors de déplacements exceptionnels.

Ainsi, l'Exposition Universelle de Paris justifia des affrètements en 1937... Citons aussi un concours agricole à Moulins, en 1948, où l'on s'entassa tellement dans l'autorail De Dion que le plancher céda en gare de Saint-Menoux !

Il faut aussi noter que Bourbon-l'Archambault, station thermale aux eaux réputées contre les rhumatismes, entrainait un trafic voyageurs appréciable (fig. 5).

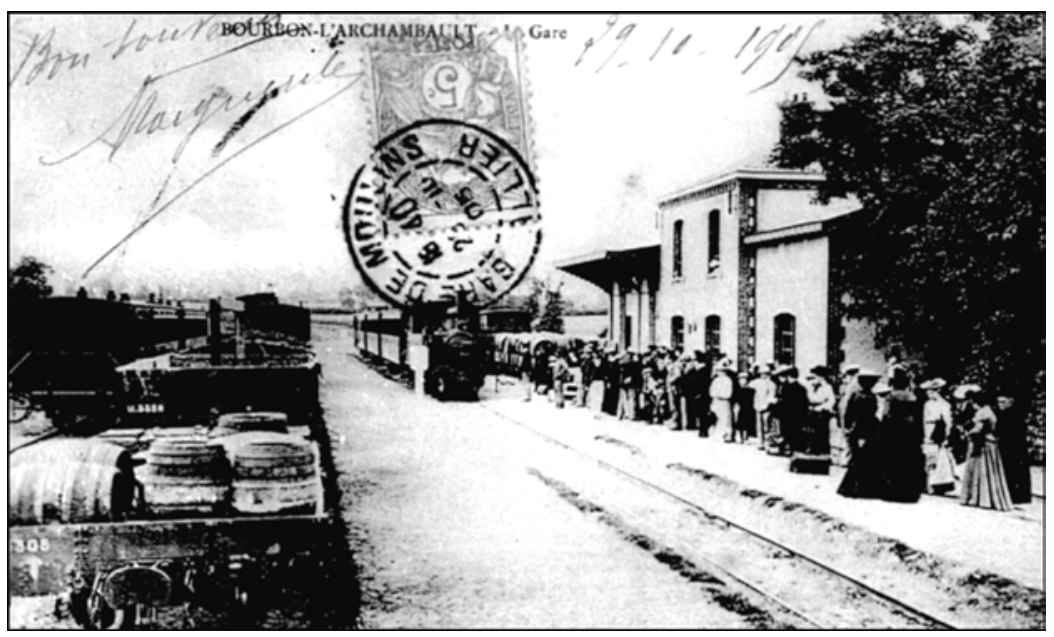

Figure 5 : Gare de Bourbon-l'Archambault. Curistes regagnant Moulins. 1910. Coll. P. Alajoinine 
En revanche, l'eau de cette station était trop calcaire pour les chaudières de locomotives! Depuis Cosne, un wagon citerne de 10 tonnes devait en transporter et être vidé dans un puits aménagé à cet effet.

\section{Les ateliers de Cosne}

Mais c'est l'implantation des ateliers de réparation et de transformation qui a surtout marqué notre localité (fig. 6). Destinés à l'ensemble du réseau économique du Centre, il arrivait que du matériel de la Nièvre y soit traité, bien que la jonction projetée, Moulins-Decize-Saxi-Bourdon, sur la ligne Nevers-Corbigny, n'ait jamais été réalisée.

Là aussi, la municipalité de l'époque sut saisir l'opportunité, supplantant les candidatures de Sancoins et de Montmarault.

Dès novembre 1887, la Société économique prenait possession des terrains. Grave dilemme : les ateliers étaient-ils destinés à la ligne d'intérêt local Moulins-Cosne ou à la ligne d'intérêt général SancoinsLapeyrouse ? Dans l'incertitude, les vendeurs de terrains, toujours impayés un an après, menaçaient de détruire les travaux réalisés! La commune régla le litige, à la hâte, en s'impliquant financièrement.

L'activité débuta en 1892 et, rapidement, la surface occupée atteignit $10000 \mathrm{~m}^{2}$. Dès 1893, un premier agrandissement de $640 \mathrm{~m}^{2}$ s'imposa, dont le financement, cette fois, fut réparti par moitié entre l'État et le département de l'Allier.

En 1906, un deuxième agrandissement avec construction d'un bâtiment surélevé, propre à recevoir un pont transbordeur, marqua l'apogée des ateliers. Ce sont 150 ouvriers spécialisés qui œuvrèrent, aux plus beaux jours, encadrés par un ingénieur, un inspecteur traction, un chef de dépôt-ateliers et un chef magasin. L'exploitation, quant à elle, justifia un ingénieur, deux inspecteurs et, bien sûr, des chefs et employés de gare.

Ainsi, réunissant ateliers, traction, voie et exploitation, l'effectif approcha de 220 agents, recrutés aux alentours. Tous les corps de métiers furent représentés : ajusteurs, tourneurs, chaudronniers, ferreurs-wagons, forgerons, mais aussi menuisiers, peintres, serruriers, charrons, tapissiers, sans oublier les indispensables services auxiliaires. 
Revue d'histoire des chemins de fer 24-25 (printemps - automne 2001)

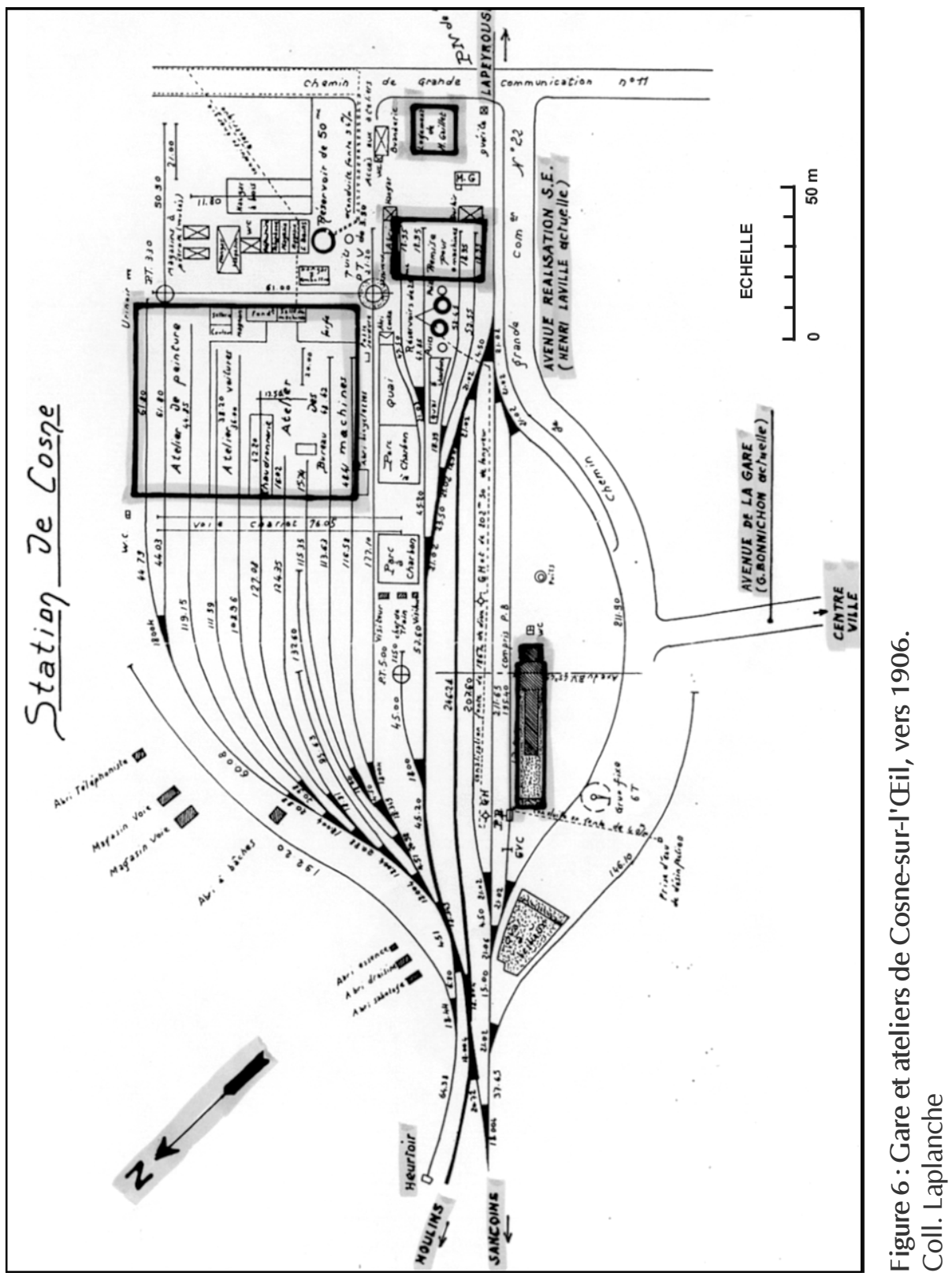


En 1936, un atelier spécial pour les automotrices De Dion dut être créé (fig. 7).
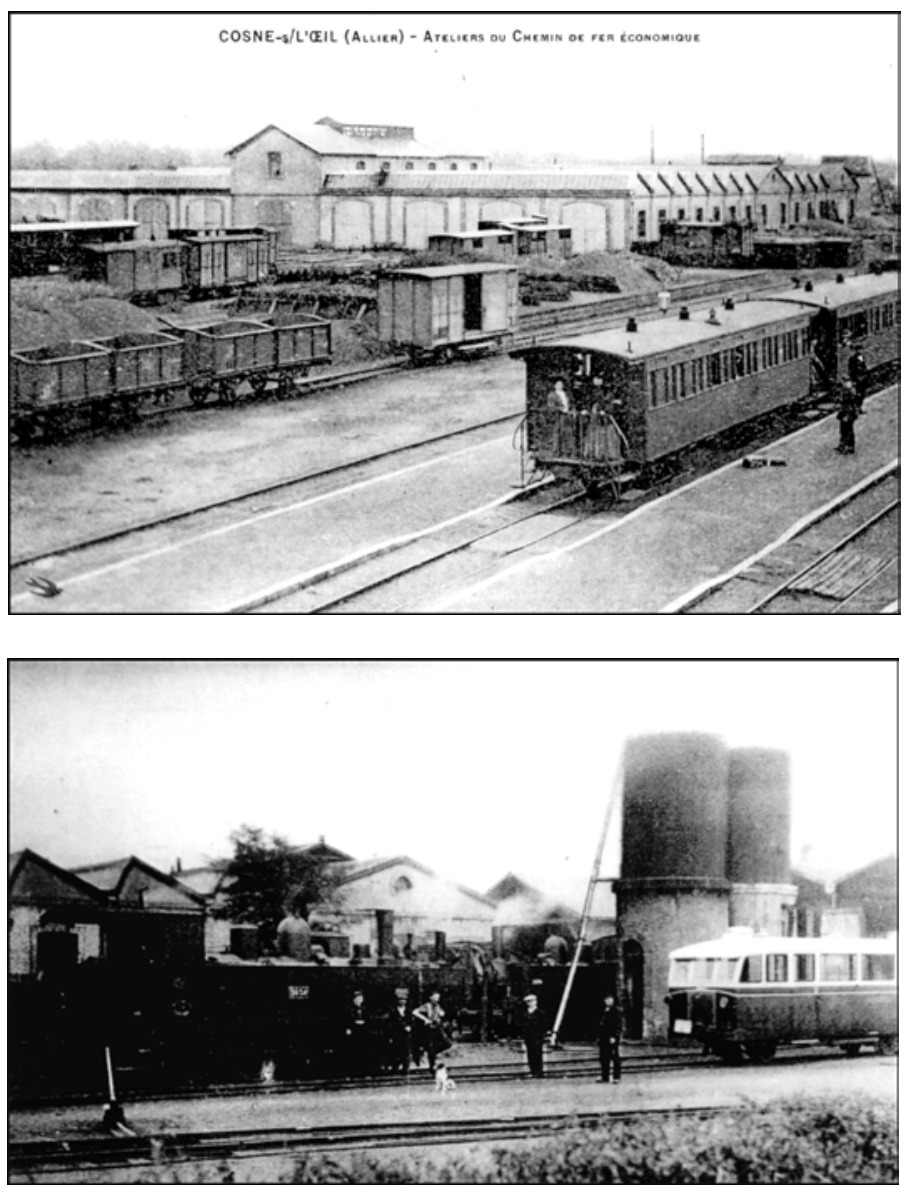

Figure 7 : Cosne-sur-l'CFil. Les ateliers.

(Haut : ateliers du Chemin de fer économique, 1908. Coll. P. Alajoinine; bas : ateliers avec 130 T et De Dion, 1943. Coll. Laplanche)

Un professionnalisme de haute qualité existait dans ces ateliers. On aimait ces locomotives dont les noms évoquaient des localités environnantes : Torterais, Bézenet.

La sirène des ateliers rythmait non seulement les mouvements de personnel, mais aussi la vie des riverains. 


\section{Incidences sur l'urbanisme}

En effet, les ateliers ne demeurèrent pas longtemps isolés. La pénurie de logements se manifesta dès leur ouverture. Résidences, commerces, artisanat nouveaux apparurent le long des rues existantes et de nouvelles voies durent rapidement être tracées (fig. 8).
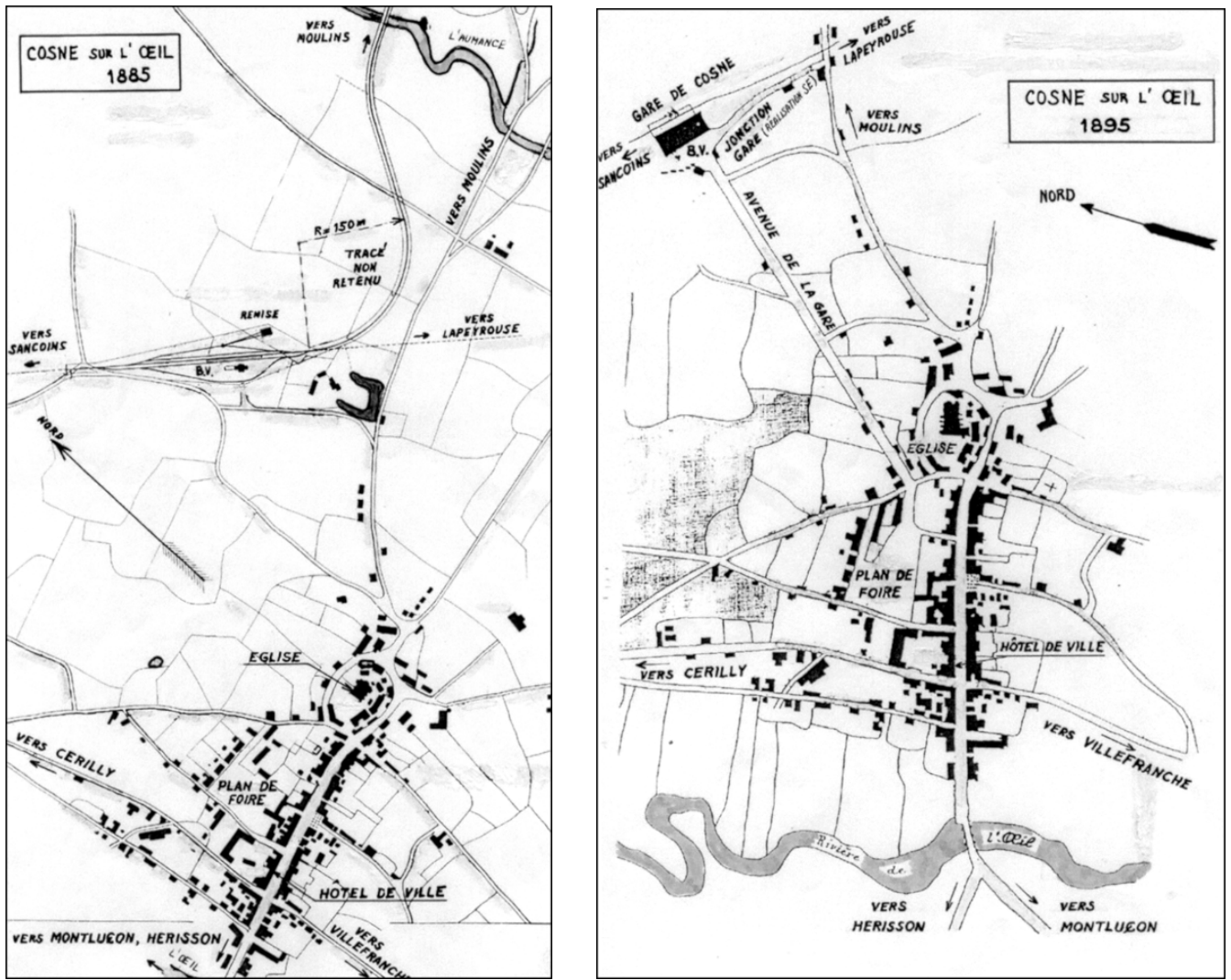

Source: Archives départementales de l'Allier

Figure 8 : Évolution de Cosne à l'arrivée du « Tacot ».

Il fallut néanmoins attendre la fin de la Première Guerre mondiale pour que des cités soient construites. Elles reçurent le nom de « Berthelier », l'un des directeurs de la Société économique. Celle-ci avait également construit trois immeubles à usage de bureaux et de logements. Le «Tacot » a manifestement entrainé un puissant développement de la localité, vers et au-delà de la gare et des ateliers. 
Enfin, l'alimentation en eau de ceux-ci, que le creusement d'un puits n'avait pu satisfaire, justifia l'édification d'un mini-barrage sur la rivière Aumance avec un bélier de refoulement.

\section{Incidences sociales}

La croissance démographique de la commune, marquée depuis 1860, s'est alors accentuée. Si la majoration de quelque 250 âmes, de 1886 à 1911, peut paraittre modeste, il faut considérer que les nouveaux emplois créés par l'«Économique » pallièrent, à Cosne, l'important exode rural de l'époque.

Sur le plan social, il est certain que le « Tacot » entraina un profond bouleversement. Dans cette localité de commerçants, artisans et agriculteurs, la composante ouvrière représenta désormais le tiers de la population et l'orientation politique s'en trouva modifiée.

La Société économique apporta elle-même un souffle social nouveau. C'était la gratuité des soins, l'assistance lors des congés de maladie, l'attribution de vêtements et de chaussures aux enfants, puis l'aide aux veuves et aux orphelins après la tragédie de 1914-1918, sans oublier la mise à disposition de jardins ouvriers.

Cosne allait cependant être le théâtre de mouvements revendicatifs, notamment en 1920.

La vie associative se développa spectaculairement, grâce au personnel du chemin de fer, tant sur le plan sportif (cyclisme, pêche, puis football en particulier), que culturel, comme la musique.

La distribution s'illustra aussi par la création d'un important magasin coopératif.

\section{Les effets de la Grande Guerre}

Revenons au « Tacot » lui-même, dont le quasi-monopole assura la relative prospérité jusqu’à la Grande Guerre, bien que, hormis sur la ligne Moulins-Cosne, sa rentabilité ne fut jamais assurée dans l'Allier.

Un lourd tribut fut apporté à la bataille de Verdun par le transfert de matériel, surtout de traction, sur le «Meusien » ainsi que sur la ligne Toul-Thiaucourt.

Comme partout, des femmes se substituèrent aux hommes mobilisés et permirent le maintien d'une exploitation réduite. Parallèlement, des "territoriaux », trop âgés pour être engagés au front, assurèrent la garde des installations. 
Le conflit achevé, il fallut remettre en état du matériel naguère utilisé dans le Nord de la France. Les ateliers de Cosne connurent alors une activité très intense et du personnel fut détaché de la Somme, pour grossir les effectifs.

Clin d'œil des passionnés du rail, c'est sur le Chemin de fer touristique de la Baie de Somme que les Bourbonnais peuvent retrouver aujourd'hui l'ambiance de leur petit train disparu! Et surtout, la « voiture-salon des officiels » de l'Allier y a heureusement trouvé refuge.

\section{Déclin}

Mais, après cette dure période, l'automobile allait inexorablement prendre l'avantage en cette zone rurale. En dépit du confort et de vitesses commerciales accrus, apportés par les De Dion, accueillis sous le vocable de «michelines », la fermeture de toutes les lignes métriques de l'Allier, hors Vichy-Lavoine-Laprugne et les deux desservant Cosne, intervint le 15 juin 1939. Commentry-Marcillat avait déjà disparu le $1^{\text {er }}$ janvier 1933.

Bien sûr, l'activité des ateliers s'en trouva réduite, des reclassements furent nécessaires et les employés de plus de cinquante ans furent mis en pré-retraite. Et pourtant, que de services rendirent MoulinsCosne, Sancoins-Lapeyrouse et le réseau du Cher, encore intact, durant la Seconde Guerre mondiale !

Le franchissement de la ligne de démarcation, entre zone libre et occupée, à Moulins surtout, fut assorti de nombreuses dissimulations aux yeux de l'ennemi d'alors. Des agents furent engagés afin de les soustraire au travail obligatoire en Allemagne. Les vaillantes «130 Schneider» furent utilisées à la limite de leurs possibilités. Aux ateliers, on faisait assaut d'imagination pour assurer au matériel un entretien convenable, en dépit du manque de produits essentiels.

Ce furent les autorails qui s'en tirèrent le mieux. Le gazogène put leur être adapté, favorisé par les ressources de l'important massif forestier des environs.

Le conflit terminé, l'automobile reprit sa suprématie et le trafic déclina rapidement. Bien que la ligne Moulins-Cosne demeurât la plus active, la suppression s'amorça par celle du trafic voyageurs, le $1^{\text {er }}$ janvier 1950. Six mois plus tard, les trains de marchandises cessèrent de rouler entre Buxières et Cosne.

Enfin, le $1^{\text {er }}$ janvier 1951, ce fut la fin de Sancoins-Villefranche, précédant de six mois la fermeture de l'ensemble du réseau du Cher. En dernier, les ateliers de Cosne construisirent des locotracteurs à partir 
de châssis d'anciennes locomotives, comme en témoigne cette émouvante photographie (fig. 9). Hélas, ils ne connurent pas l'heureuse destinée des ateliers C.F.D. de Montmirail.

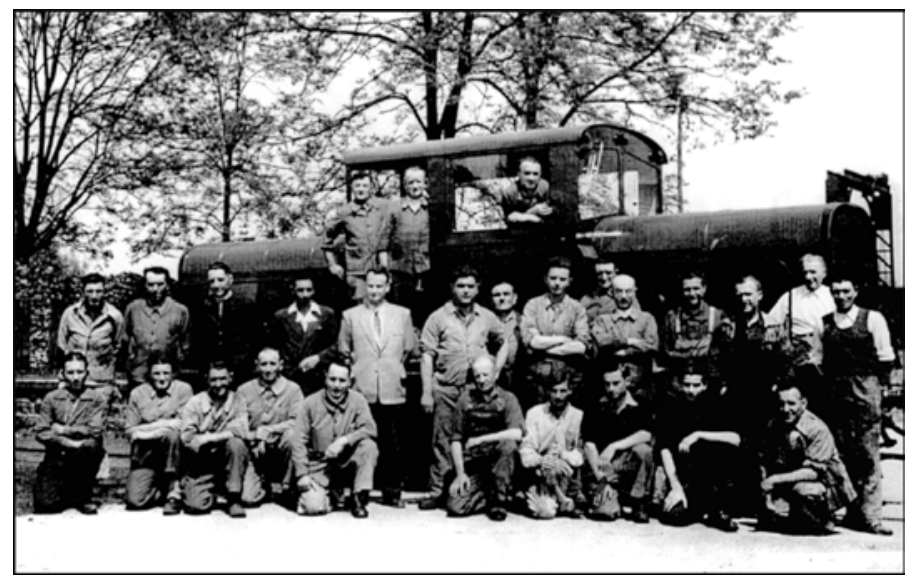

Figure 9 : Locotrateur réalisé à Cosne, 1950.

Coll. Couture

\section{Vestiges}

Que reste-t-il, de nos jours?

$\mathrm{Si}$ « La Coop » et « L'Amicale », harmonie où le personnel S.E. était prédominant, ont disparu, l'élan donné à la vie associative a perduré. L'orientation politique, imprimée dans la commune par le personnel des ateliers, est demeurée dans les esprits.

Mille mètres de la ligne de Sancoins sont devenus promenade piétonne, les cités ont toujours bon aspect et la tour du bélier se dresse encore fièrement, à deux pas de son barrage et d'un pont ferroviaire. Des logements de garde-barrière, mais aussi des gares comme celles de Bourbon-l'Archambault, Sancoins, Lurcy-Lévy, demeurent très représentatives de l'architecture de la Société économique (fig. 10). Mais ce sont surtout les ateliers qui constituent un élément d'archéologie industrielle remarquable, tant par leur ampleur, $4000 \mathrm{~m}^{2}$ couverts, que par leur aspect extérieur (fig. 11). 
Revue d'histoire des chemins de fer 24-25 (printemps - automne 2001)

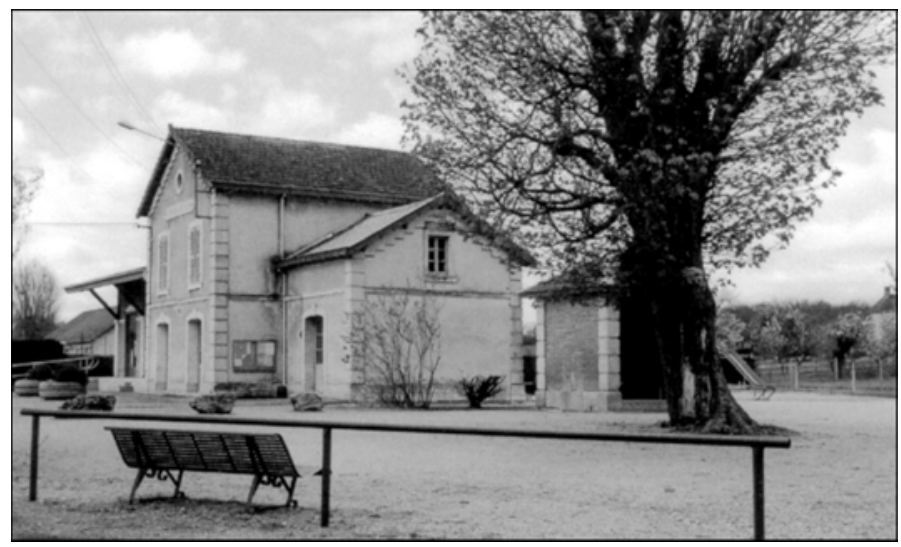

Figure 10 : Gare de Lurcy-Lévy, 2000.

Coll. R. Courtaud
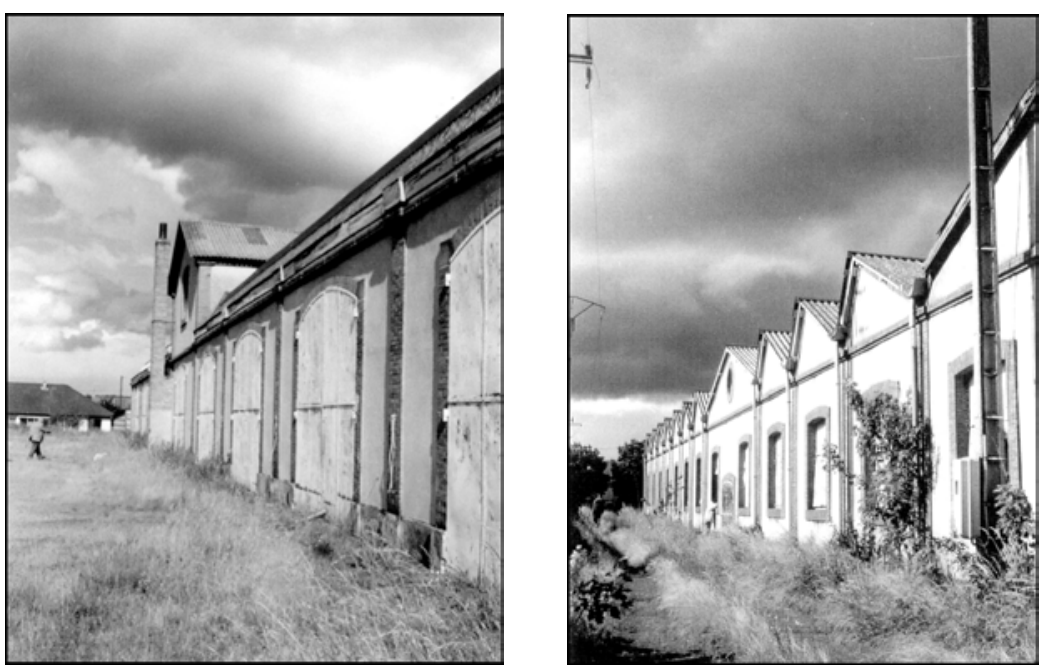

Figure 11 : État des ateliers de Cosne (façades nord et ouest) en 2001. Cl. G. Santoni 
Notre localité souhaite ardemment préserver ce témoin exceptionnel des chemins de fer «secondaires » et, plus particulièrement, de l'Économique.

Voici deux ans, notre association a présenté une demande de classement auprès de la Région Auvergne, sans suite pour l'instant. Notre principal souci est que, si un quart de la superficie, l'ancien dépôt notamment, est propriété de la commune, le reste appartient à un agriculteur qui, en parfaite légalité, peut l'anéantir à tout moment. Une tentative de rachat pourrait être envisagée.

Monsieur le maire et conseiller général de Cosne y est favorable. Mais comment plaider la cause en haut lieu si une utilisation concrète des locaux n'est pas proposée ? Tel est notre douloureux dilemme, que certain projet de musée au niveau de l'arrondissement de Montluçon, n’a pu faire évoluer. Bien sûr, si au sein de l'auditoire hautement motivé par l'histoire des chemins de fer dits « secondaires ", auquel nous avons l'honneur de nous adresser aujourd'hui, une proposition d'utilisation de ces anciens ateliers se manifestait, nous serions très heureux d'en débattre.

Enfin, si un pèlerinage parmi les vestiges de notre regretté « Tacot » vous tentait, flânant ainsi dans notre paisible et pittoresque Bourbonnais, sachez que vous seriez toujours les bienvenus à Cosne d'Allier. 